\title{
Artificial Pancreas Using a Personalized Rule-Based Controller Achieves Overnight Normoglycemia in Patients with Type 1 Diabetes
}

\author{
Ismael Capel, MD, Mercedes Rigla, PhD, Gema García-Sáez, PhD, 2,3 \\ Agustín Rodríguez-Herrero, $\mathrm{PhD}^{3}$ Belén Pons, MSc, David Subías, MD, \\ Fernando García-García, MSc, ${ }^{2,3}$ Maria Gallach, RN, ${ }^{1}$ Montserrat Aguilar, RN, \\ Carmen Pérez-Gandía, MSc, ${ }^{2,3}$ Enrique J. Gómez, PhD, 2,3 \\ Assumpta Caixàs, $\mathrm{PhD}^{1}$, and M. Elena Hernando, $\mathrm{PhD}^{2,3}$
}

\begin{abstract}
Objective: This study assessed the efficacy of a closed-loop (CL) system consisting of a predictive rule-based algorithm (pRBA) on achieving nocturnal and postprandial normoglycemia in patients with type 1 diabetes mellitus (T1DM). The algorithm is personalized for each patient's data using two different strategies to control nocturnal and postprandial periods. Research Design and Methods: We performed a randomized crossover clinical study in which 10 T1DM patients treated with continuous subcutaneous insulin infusion (CSII) spent two nonconsecutive nights in the research facility: one with their usual CSII pattern (open-loop [OL]) and one controlled by the pRBA (CL). The CL period lasted from 10 p.m. to 10 a.m., including overnight control, and control of breakfast. Venous samples for blood glucose (BG) measurement were collected every $20 \mathrm{~min}$.

Results: Time spent in normoglycemia (BG, 3.9-8.0 mmol/L) during the nocturnal period (12 a.m.-8 a.m.), expressed as median (interquartile range), increased from $66.6 \%(8.3-75 \%)$ with OL to $95.8 \%(73-100 \%)$ using the CL algorithm $(P<0.05)$. Median time in hypoglycemia $(\mathrm{BG},<3.9 \mathrm{mmol} / \mathrm{L})$ was reduced from $4.2 \%(0-21 \%)$ in the OL night to $0.0 \%(0.0-0.0 \%)$ in the $\mathrm{CL}$ night $(P<0.05)$. Nine hypoglycemic events $(<3.9 \mathrm{mmol} / \mathrm{L})$ were recorded with $\mathrm{OL}$ compared with one using CL. The postprandial glycemic excursion was not lower when the CL system was used in comparison with conventional preprandial bolus: time in target (3.9-10.0 mmol/L) 58.3\% (29.1-87.5\%) versus 50.0\% (50-100\%).

Conclusions: A highly precise personalized pRBA obtains nocturnal normoglycemia, without significant hypoglycemia, in T1DM patients. There appears to be no clear benefit of CL over prandial bolus on the postprandial glycemia.
\end{abstract}

\section{Introduction}

$\mathbf{T}$ YPE 1 DiABETES Mellitus (T1DM) implies a lifethreatening absolute insulin deficiency. Since the 1920s substitution of the hormone using the subcutaneous route has allowed for the disappearance of ketosis and acute symptoms of hyperglycemia and, in recent decades, has led to a significant reduction in the risk of onset and progression of the long-term microvascular complications thanks to intensified insulin treatments. ${ }^{1}$ However, many imperfections of this treatment, such as risk of severe hypoglycemia, ${ }^{2}$ constitute a barrier to achieving an optimal blood glucose (BG) control in most patients, even when a continuous subcutaneous insulin infusion (CSII) system is used. Some biological solutions for T1DM, such as immunomodulation at disease onset or islet transplantation, are promising, but, unfortunately, the results they yield are still too poor for near-future clinical application. ${ }^{3}$ Another way of improving current therapies is the integration of real-time continuous glucose monitoring (CGM), a technique that has been refined in recent years, ${ }^{4}$ and the CSII system, which executes the optimal insulin dose proposed by a computerized controller, implementing an

\footnotetext{
${ }^{1}$ Endocrinology and Nutrition Department, Parc Taulí Sabadell University Hospital, Autonomous University of Barcelona, Sabadell, Barcelona, Spain.

${ }^{2}$ Networking Research Centre for Bioengineering, Biomaterials and Nanomedicine, Madrid, Spain.

${ }^{3}$ Bioengineering and Telemedicine Group, Polytechnical University of Madrid, Madrid, Spain.

This study is registered at ClinicalTrials.gov with trial registration number NCT01614496.
} 
artificial pancreas or artificial $\beta$-cell. Closed-loop (CL) is a specific control engineering term that has also been widely used to describe the integration of these technologies for glucose control. Using the same terminology, open-loop (OL) refers to scenarios where conventional CSII therapy is used.

When using the subcutaneous route, for both measurement and insulin administration, CL strategies have to fight against well-identified difficulties, the most challenging probably being the delay in rapid glucose change detection and the lengthy time needed for subcutaneously administered insulin to act. ${ }^{5}$

The most widely used strategies for CL controllers have been focused on proportional integral derivative control ${ }^{6,7}$ and model predictive control. ${ }^{8,9}$ Other approaches use an inverse controller ${ }^{10}$ or imitate the lines of reasoning of diabetes caregivers, using methods such as fuzzy logic control ${ }^{11}$ or rule-based algorithms. ${ }^{12}$

In the last few years, some clinical experience with CL control algorithms has yielded favorable results in regulating BG levels in subjects with T1DM, both in-clinic ${ }^{6-9,13}$ or very closely supervised conditions. ${ }^{11}$ The percentage of time in BG target range was generally defined as the main goal. For studies including postprandial periods, although many of them included postprandial time in their overall results, better outcomes were obtained in those studies in which a preprandial manual bolus was used. ${ }^{14}$ Studies focusing on nocturnal periods have achieved better results: the percentage of time ranging from 3.9 to $8.0 \mathrm{mmol} / \mathrm{L}$ varied roughly from $70 \%$ to $80 \%{ }^{8,9}$ In the light of these results, it seems reasonable to assume that nocturnal control will become the first step toward CL strategy implementation in real life.

Our group has developed a glucose controller using a predictive rule-based algorithm (pRBA) that takes into account the instant value of glucose indicated by CGM, the predicted rate of change (pROC), and the nominal basal insulin. In order to assess the efficacy of the pRBA, measured as time spent in BG target values (3.9-8.0 $\mathrm{mmol} / \mathrm{L})$, and safety, measured as the incidence of hypoglycemic events, we performed a crossover clinical trial comparing an OL night, using CSII therapy, with a CL night.

\section{Research Design and Methods}

\section{The $p R B A$ system}

The pRBA has been designed following the controlto-range concept ${ }^{15,16}$ and complemented by a safety control module to prevent insulin overdose, similar to other safety systems. ${ }^{10,17}$ The pRBA is a hybrid system that requires a partial premeal insulin administration after considering the amount of carbohydrates of each meal intake. The algorithm uses two different strategies to control basal or postmeal periods, with the mealtime being the reference for switching between them.

The algorithm is personalized for each patient using the subject's adjusted nominal insulin, personal parameters, such as the correction factor or the insulin-to-carbohydrates ratio, and some physical characteristics, such as the patient's weight. Information for personalizing the pRBA is extracted from the insulin treatment and data recorded in the patient's daily logbook with regard to BG levels, food intake, and other significant events. The pRBA requires no specific subject model, and it uses rules with explicit knowledge.
The control-to-range module operates according to the current glucose value and the pROC calculated with a glucose predictor based on an artificial neural network. ${ }^{18}$ The predictor is trained with CGM dataset sensor files and takes into account inter- and intrapatient variability. The time horizon for glucose prediction is $30 \mathrm{~min}$.

The goal of the control-to-range module is to achieve glucose values between $4.4 \mathrm{mmol} / \mathrm{L}$ and $7.8 \mathrm{mmol} / \mathrm{L}$ and to assure glucose stability, defined as pROC within $\pm 0.28 \mathrm{mmol} /$ $(\mathrm{L} \cdot \min )(5 \mathrm{mg} /[\mathrm{dL} \cdot \mathrm{min}])$. The pRBA control-to-range module provides as output a parameter $n$, which is applied to the nominal basal insulin of each patient and, when required, a correction bolus.

The control-to-range module is complemented by a safety control module that modifies the final proposed insulin delivery based on the insulin on board, which takes into account both the insulin pharmacodynamics and the CSII system constraints. The safety control rules limit the maximum insulin infusion and suspend the insulin delivery at a sensor glucose level lower than $4.4 \mathrm{mmol} / \mathrm{L}$ unless the $\mathrm{pROC}$ indicates a clear increasing trend (pROC $>0.28 \mathrm{mmol} /[\mathrm{L} \cdot \mathrm{min}])$ or when, despite glucose values being within the desired range, the pROC indicates a highly fast-decreasing trend (pROC $<-0.84 \mathrm{mmol} /[\mathrm{L} \cdot \mathrm{min}])$. The time for insulin infusion suspension is limited to $2 \mathrm{~h}$, but a microbolus of the least possible insulin dose is administered every $30 \mathrm{~min}$ after suspension to avoid catheter occlusions.

The final pRBA output is calculated by combining the control-to-range and the safety control modules, thereby obtaining an insulin micro-dose at each time step (every $5 \mathrm{~min}$ ).

The pRBA was evaluated in silico before the clinical study to test its performance in different conditions. The patient population was simulated ${ }^{10}$ using the compartmental model defined previously. ${ }^{19}$

\section{Clinical study}

Ten adults with T1DM previously treated with CSII were enrolled in this randomized crossover study. Inclusion criteria were minimum age of 18 years, diabetes duration longer than 3 years, and CSII treatment for more than 1 year. Exclusion criteria were hemoglobin A1c of $>10 \%(86 \mathrm{mmol} / \mathrm{mol})$ and concomitant diseases or treatments with drugs that potentially affect glucose control. The entire study was performed at the Diabetes Day Care Center, Parc Taulí Sabadell University Hospital, Barcelona, Spain, and was approved by the local ethics board (http://www.tauli.cat/tauli/en/docencia/ recerca/recerca.htm). A written informed consent was obtained from all patients.

Patients spent two nonconsecutive nights in the hospital: one night with their usual CSII therapy (OL night) and one night controlled by the pRBA (CL night). The order of participation was determined by randomization. Two patients, one for each treatment option, were involved on each night. The CL period lasted from 10 p.m. to 10 a.m., including overnight control and control of breakfast, given at 8 a.m.

On the CL night, each patient was equipped with two CGM devices (Paradigm ${ }^{\circledR}$ REAL-Time; Medtronic, Minneapolis, $\mathrm{MN}$ ) beginning at least $24 \mathrm{~h}$ before admission. CGM devices were calibrated at home using the patient's glucose meter according to the manufacturer's recommendations. 
Patients arrived at the hospital at 6 p.m. Then, one sensor was chosen as the reference measurement for the experiment based on its reliability and accuracy, whereas the second one was kept as backup to be used in case of reference sensor failure. On patient admission, the CGM devices were calibrated using an YSI STAT Plus ${ }^{\mathrm{TM}} 2300$ glucose and lactate analyzer (YSI, Yellow Springs, OH). Dinner (Mediterranean salad, roast chicken, fries, bread, and fruit) and breakfast (white coffee, bread, ham, and cheese) meals were identical on both nights, and their composition was calculated according to each patient's habits. An intravenous cannula was inserted into each patient's antecubital vein. Blood samples were collected every $20 \mathrm{~min}$ for reference glucose measurement. Hypoglycemia was defined as a YSI reading of $<3.9 \mathrm{mmol} / \mathrm{L}$ with or without symptoms recognized by the patient as relating to hypoglycemia and was treated with 5-20 g of carbohydrate (orange juice).

At 10 p.m. the patient's CSII was switched to an Animas ${ }^{\circledR}$ 2020 (Animas Corp., West Chester, PA), chosen because it allows the fastest bolus delivery. The insulin pump was previously filled with insulin analog aspart (Novorapid ${ }^{\circledR}$; Novo Nordisk, Copenhagen, Denmark). The insulin basal infusion was set to zero when the CL period started. pRBA was initialized with each patient's data (CGM glucose, carbohydrate intake, and delivered insulin) recorded during the 10 -h period preceding the start of the CL delivery.

The sensor reading, pump operation, and the algorithmrunning were done manually by two different members of the team located in different rooms. As the algorithm was executed very frequently, only one person stayed in the room with the patient in order to avoid disturbing him or her during the night. An ad hoc local area network, not connected to the Internet, was configured to allow the researchers to communicate through videoconference software, including a chat message service. Three computers were connected to the network at the following sites: the patient's room, the algorithm area, and the YSI. An alarm clock software producing light and sound alarms was running and synchronized with all three computers. From 12 midnight to 7 a.m., the sound alarms were switched off.

The process was as follows: every $5 \mathrm{~min}, 30$ and $10 \mathrm{~s}$ before the scheduled time, the alarm clock activated an alarm, and the physician seated close to the patient read the sensor monitors (reference and backup) and wrote the two glucose values into the chat. In the algorithm area, a technician entered the reference sensor glucose value into the algorithm, which then calculated the new insulin microbolus. The technician wrote the insulin dose proposed by the algorithm into the chat, and the patient-side physician operated the pump to deliver the insulin. The whole process took around 30 s. Every 20 min, YSI values were also sent by chat from the YSI-side computer. This procedure was maintained for $12 \mathrm{~h}$. To facilitate controller supervision, all the information was available to all the researchers in real time both as numerical data and as graphs. The research team comprised two research nurses, three physicians, and three technicians.

At 7:45 a.m. a priming bolus of $50 \%$ of the usual bolus was given, and at 8 a.m. breakfast was served. The rest of the prandial insulin was administered by pRBA as required by the evolution of CGM values. At 10 a.m. the experiment finished, and patients returned to their usual CSII with the usual basal profile.
For the OL night, a single CGM sensor was inserted for at least $24 \mathrm{~h}$ before admission with data being available for the patient. Patients arrived at the research facility at 6 p.m., and, as on the CL night, dinner was served at 8 p.m., breakfast was served at 8 a.m., and patients stayed in the hospital until 10 a.m. However, on the OL night they used a sensor-augmented pump with their usual basal rates and bolus during the whole study period. Blood samples were collected, as on the CL night, for venous glucose measurement, every 40 min from 12 a.m. to 8 a.m. and every $20 \mathrm{~min}$ for the rest of the period.

\section{Statistics}

The statistical analysis plan was designed in advance. The primary outcome was time spent in normoglycemia (3.9$8.0 \mathrm{mmol} / \mathrm{L}$ ) during the overnight period (12 a.m.-8 a.m.). Secondary outcomes were percentage of time and number of episodes of hypoglycemia (BG level, $<3.9 \mathrm{mmol} / \mathrm{L}$ ) and variability parameters (interquartile range, $\mathrm{SD}$, Low $\mathrm{BG}$ Index, High BG Index, and BG Risk Index). ${ }^{20}$ All the results are expressed as mean $\pm \mathrm{SD}$ or median (interquartile range) values. For calculating percentiles, Tukey's method was used. Statistical comparison between OL and CL was done using the nonparametric test for paired samples (Wilcoxon test). Analyses were carried out with SPSS version 20 software (IBM, Armonk, NY).

\section{Results}

All 10 patients (five women; mean \pm SD age, $46.7 \pm 10.82$ years old; mean $\pm S D$ diabetes duration, $18.0 \pm 5.7$ years; mean \pm SD body mass index, $25.7 \pm 3.2 \mathrm{~kg} / \mathrm{m}^{2}$; mean \pm SD hemoglobin A1c, $7.1 \pm 0.8 \%$ [55 $\pm 8.7 \mathrm{mmol} / \mathrm{mol}]$ ) completed the study. Carbohydrate content of the dinner was $57.0 \pm 16.3 \mathrm{~g}$, and that of the breakfast was $41.0 \pm 12 \mathrm{~g}$. All the insulin doses proposed by the algorithm were checked by an endocrinologist and actually administered. Individual characteristics of the patients are shown in Table 1.

\section{Accuracy of CGM}

The sensors chosen for the CL part of the study showed an acceptable accuracy (median absolute relative difference, $12.6 \%$ [4.8-26.5\%]). Clarke error grid analysis ${ }^{21}$ showed $79.4 \%$ in Zone A, $18.7 \%$ in Zone B, and 1.8\% in Zone D. Continuous error grid analysis ${ }^{22}$ showed $81.4 \%$ of values in Zone A for both rate and point-error grid analysis regarding euglycemia. However, this percentage decreased to $22.2 \%$ for hypoglycemia ( $\leq 3.9 \mathrm{mmol} / \mathrm{L}$ ) and $58.6 \%$ for hyperglycemia $(>10 \mathrm{mmol} / \mathrm{L})$. The second sensor was not used to run the algorithm at any time. Occasionally, there were very short periods of reference sensor signal loss, but these were always shorter than $10 \mathrm{~min}$. On these occasions the previous reference sensor value was used.

\section{Overnight period}

From starting CL to breakfast (10 p.m.-8 a.m.). Starting BG level was similar in the OL and CL sessions (8.9 \pm 4.1 vs. $8.7 \pm 5.1 \mathrm{mmol} / \mathrm{L})$. No statistical differences were found in the overnight mean BG between OL and CL nights (7.8 \pm 2.3 vs. $6.8 \pm 1.9 \mathrm{mmol} / \mathrm{L})$, although the range of BG was tighter (5.4$12.3 \mathrm{mmol} / \mathrm{L}$ for OL nights vs. $4.9-10.6 \mathrm{mmol} / \mathrm{L}$ for CL nights) (Fig. 1). Percentage of time in normoglycemia (3.9-8 mmol/L) 
Table 1. Individual Data of All Patients

\begin{tabular}{|c|c|c|c|c|c|c|c|c|c|c|c|}
\hline & \multirow{2}{*}{$\begin{array}{c}\text { Total/ } \\
\text { mean } \pm S D\end{array}$} & \multicolumn{10}{|c|}{ Subject identification } \\
\hline & & 1 & 2 & 3 & 4 & 5 & 6 & 7 & 8 & 9 & 10 \\
\hline $\operatorname{Sex}(M / F)$ & $5 / 5$ & $\mathrm{~F}$ & $\mathrm{~F}$ & M & M & M & M & M & $\mathrm{F}$ & $\mathrm{F}$ & $\mathrm{F}$ \\
\hline Age (years) & $46.7 \pm 10.8$ & 64 & 35 & 59 & 39 & 43 & 58 & 51 & 39 & 46 & 33 \\
\hline Height (m) & $1.7 \pm 0.1$ & 1.64 & 1.60 & 1.65 & 1.81 & 1.90 & 1.70 & 1.69 & 1.64 & 1.66 & 1.67 \\
\hline Weight (kg) & $74.5 \pm 15.1$ & 64.4 & 64.9 & 75.7 & 75 & 110.6 & 65.9 & 89.3 & 64.4 & 62 & 72.6 \\
\hline $\mathrm{BMI}\left(\mathrm{kg} / \mathrm{m}^{2}\right)$ & $25.7 \pm 3.2$ & 23.9 & 25.3 & 27.8 & 22.9 & 30.6 & 22.8 & 31.2 & 23.9 & 22.5 & 26.0 \\
\hline HbA1c (\%) & $7.1 \pm 0.8$ & 7.6 & 5.6 & 7.8 & 8.2 & 7.3 & 7.9 & 6.8 & 6.2 & 7.3 & 7.1 \\
\hline $\mathrm{HbA1c}(\mathrm{mmol} / \mathrm{mol})$ & $55.0 \pm 8.7$ & 60 & 38 & 62 & 66 & 56 & 63 & 51 & 44 & 56 & 54 \\
\hline TDD (U) & $43.8 \pm 17.9$ & 29.7 & 36 & 38 & 49 & 52 & 44 & 90 & 29.4 & 36.2 & 33.5 \\
\hline Duration of diabetes (years) & $18.0 \pm 5.7$ & 18 & 9.5 & 17 & 12 & 13 & 16 & 21 & 28 & 24 & 22 \\
\hline Duration insulin pump (years) & $6.9 \pm 2.4$ & 4 & 5 & 11 & 5 & 5 & 8 & 8 & 9 & 9 & 5 \\
\hline Dinner size ( $g$ of carbohydrates) & $57.0 \pm 16.3$ & 60 & 40 & 50 & 60 & 60 & 90 & 60 & 30 & 70 & 50 \\
\hline Breakfast size (g of carbohydrates) & $41.0 \pm 12$ & 50 & 30 & 20 & 40 & 60 & 40 & 50 & 30 & 50 & 40 \\
\hline \multicolumn{12}{|l|}{ Open loop } \\
\hline Mean BG overnight period (mmol/L) & $7.79 \pm 2.3$ & 9.81 & 5.58 & 9.05 & 6.02 & 5.37 & 9.25 & 5.6 & 7.87 & 7.08 & 12.31 \\
\hline \multicolumn{12}{|c|}{ Time at glucose level (\%) overnight period } \\
\hline$<3.9 \mathrm{mmol} / \mathrm{L}$ & $7.3 \pm 9.1$ & 0 & 20 & 0 & 20 & 6.7 & 6.7 & 20 & 0 & 0 & 0 \\
\hline $3.9-8 \mathrm{mmol} / \mathrm{L}$ & $49.7 \pm 32.6$ & 3.3 & 80 & 6.7 & 60 & 93.3 & 60 & 70 & 50 & 66.7 & 6.7 \\
\hline $8-10 \mathrm{mmol} / \mathrm{L}$ & $24.0 \pm 24.9$ & 50 & 0 & 76.6 & 20 & 0 & 6.7 & 10 & 43.3 & 13.3 & 20 \\
\hline$>10 \mathrm{mmol} / \mathrm{L}$ & $19.0 \pm 24.4$ & 46.7 & 0 & 16.7 & 0 & 0 & 26.6 & 0 & 6.67 & 20 & 73.3 \\
\hline \multicolumn{12}{|l|}{ Closed loop } \\
\hline Mean BG overnight period $(\mathrm{mmol} / \mathrm{L})$ & $6.82 \pm 1.9$ & 5.94 & 5.52 & 9.66 & 10.58 & 5.58 & 4.94 & 7.13 & 5.87 & 5.18 & 7.76 \\
\hline \multicolumn{12}{|c|}{ Time at glucose level (\%) overnight period } \\
\hline$<3.9 \mathrm{mmol} / \mathrm{L}$ & $0.7 \pm 2.1$ & 0 & 6.7 & 0 & 0 & 0 & 0 & 0 & 0 & 0 & 0 \\
\hline $3.9-8 \mathrm{mmol} / \mathrm{L}$ & $79.0 \pm 28$ & 100 & 93.3 & 13.3 & 53.3 & 100 & 93.3 & 70 & 100 & 96.7 & 70 \\
\hline $8-10 \mathrm{mmol} / \mathrm{L}$ & $10.7 \pm 17.5$ & 0 & 0 & 53.4 & 6.7 & 0 & 6.7 & 30 & 0 & 3.3 & 6.7 \\
\hline$>10 \mathrm{mmol} / \mathrm{L}$ & $9.6 \pm 16$ & 0 & 0 & 33.3 & 40 & 0 & 0 & 0 & 0 & 0 & 23.3 \\
\hline
\end{tabular}

BG, blood glucose; BMI, body mass index; F, female; HbA1c, hemoglobin A1c; M, male; TDD, total daily dose.

was lower during OL versus CL (median [interquartile range], $60.0 \%$ [17-69\%] vs. 93.3\% [70-99\%], respectively; $P<0.05$ ) (Fig. 2). Control variability grid analysis ${ }^{23}$ showed eight patients in Zones $\mathrm{A}+\mathrm{B}$ for CL but only four patients for OL nights.

From midnight to 8 a.m. No difference in mean BG level was found between OL and CL nights (7.29 \pm 2.5 vs. $6.4 \pm 1.6 \mathrm{mmol} / \mathrm{L}$; difference not significant). Percentage of time in target was clearly higher for CL nights (OL vs. CL, $66.6 \%[8.3-75 \%]$ vs. $95.8 \%$ [73-100\%]; $P<0.05)$. Nine hypo- glycemic events were recorded using OL, but only one episode $(=3.3 \mathrm{mmol} / \mathrm{L})$ was observed using CL. Percentage of time with BG values $<3.9 \mathrm{mmol} / \mathrm{L}$ was $4.2 \%(0-21 \%)$ for OL but $0.0 \%(0-0 \%)$ for $\mathrm{CL}(P<0.05)$.

Glucose stability (12 a.m.-8 a.m.). The SD tended to decrease with CL (from $1.48 \pm 0.67 \mathrm{mmol} / \mathrm{L}$ to $0.92 \pm$ $0.44 \mathrm{mmol} / \mathrm{L} ; P=0.08)$. After $10 \mathrm{~h}$ of $\mathrm{pRBA}$ action a convergence between all subjects in a near-normoglycemic point was observed (OL vs. CL: 10 p.m., 8.0 [3.97] vs. CL 7.2 [2.86] mmol/ L; 8 a.m., 6.03 [2.05] vs. 5.22 [1.05] mmol/L) (Fig. 1).

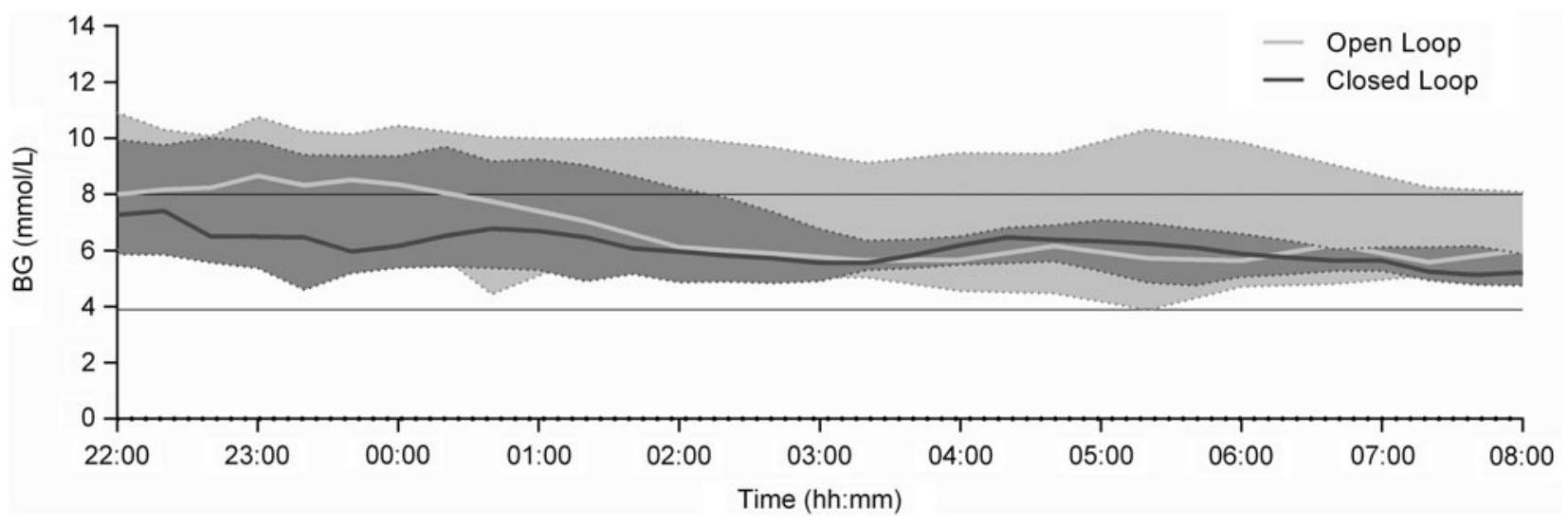

FIG. 1. Median and interquartile range of blood glucose (BG) values (YSI) during the overnight period. After $10 \mathrm{~h}$ of predictive rule-based algorithm action a convergence between all subjects in a near-normoglycemic point was observed. 


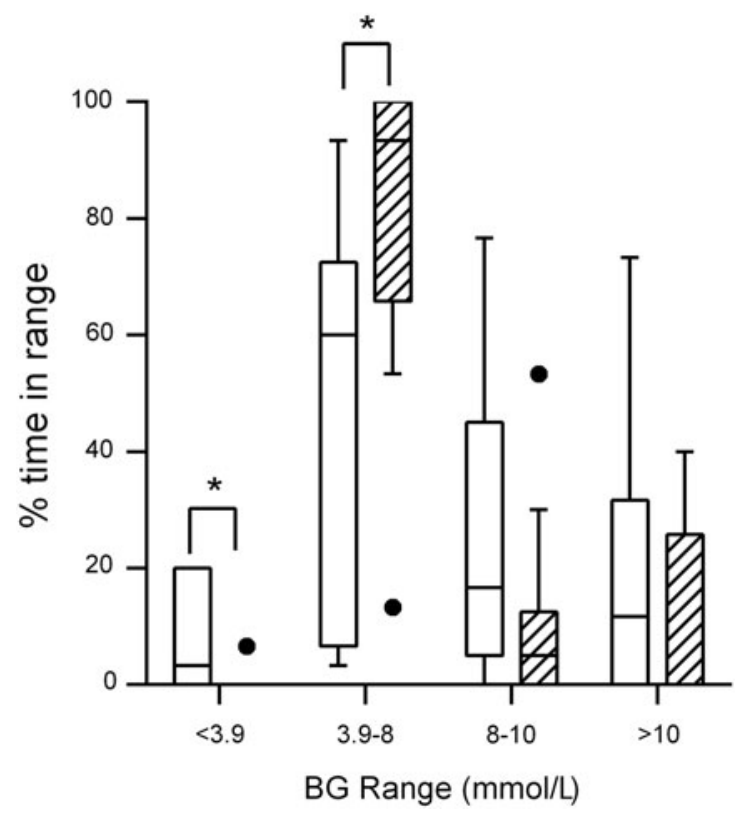

FIG. 2. Percentage of time in different blood glucose (BG) ranges corresponding to the overnight period (10 p.m.-8 a.m.): open loop (white boxes) and closed loop (striped boxes). *Difference statistically significant at $P<0.05$. Black circles correspond to outliers. Percentage of time in target was clearly higher with closed loop. Percentage of time with BG values $<3.9 \mathrm{mmol} / \mathrm{L}$ was significantly lower with closed loop.

Mean Low BG Index decreased during CL (2.18 vs. 1.09), as did High BG Index (3.95 vs. 1.60). Reduction in the BG Risk Index (6.13 to 2.70$)$ achieved statistical significance $(P<0.05)$. CGM glucose values in some patients showed an unexpected extreme stability that was confirmed by the YSI measurements: Patient 1 remained for $115 \mathrm{~min}$ between 6.0 and $6.11 \mathrm{mmol} / \mathrm{L}$, Patient 4 had sensor glucose values between 5.66 and $5.77 \mathrm{mmol} / \mathrm{L}$ for $125 \mathrm{~min}$, Patient 5 also remained for $205 \mathrm{~min}$ with sensor glucose values between 5.11 and $5.44 \mathrm{mmol} / \mathrm{L}$, and Patient 9 had sensor values between 6.66 and $6.77 \mathrm{mmol} / \mathrm{L}$ for $110 \mathrm{~min}$. This phenomenon was not observed in any patient with OL.

\section{Insulin doses}

A comparison between insulin doses actually administered (downloaded from the pump) and insulin microbolus proposed by the algorithm revealed no omissions or discrepancies.

Total insulin dose using OL was similar to the insulin dose given with $\mathrm{CL}(0.28 \pm 0.09$ vs. $0.23 \pm 0.08 \mathrm{IU} / \mathrm{kg})$, in which 144 boluses (minimum 0.05 units) were administered to each patient.

\section{Postprandial period (8 a.m.-10 a.m.)}

In total, $41 \pm 12 \mathrm{~g}$ of carbohydrates was supplied for breakfast. In CL conditions, a meal priming bolus was administered $15 \mathrm{~min}$ before ingestion (3.2 $\pm 1.7 \mathrm{IU})$. In OL, patients decided the timing for the insulin bolus based on their CGM and BG measurements. No statistical differences between CL and conventional insulin pump therapy were found in the mean BG level during the postprandial period (OL vs. CL, $8.7 \pm 3.1$ vs. $8.3 \pm 2.3 \mathrm{mmol} / \mathrm{L})$, the percentage of time in target (3.9-10.0 mmol/L) (OL vs. CL, 58.3\% [29.1-87.4\%] vs. $50.0 \%[50-100 \%])$, and the area under the curve of BG values (OL vs. CL, $1,052.7 \pm 347.5$ vs. $1,028.7 \pm 312.8 \mathrm{mmol} / \mathrm{L} \cdot \mathrm{min}$ ). Using the algorithm control, glycemic excursions seemed to be symmetrical with a maximum around 90 min after the meal intake (Fig. 3). However, with the standard premeal bolus, postprandial glycemic curves were more erratic, having oscillations and different peak times among patients.

\section{Conclusions}

This randomized crossover study has demonstrated that a CL system with a pRBA achieves normoglycemia more than 95\% of the time during the nocturnal period without increasing the risk of hypoglycemia. Postprandial results, although not inferior to those obtained with a standard premeal bolus, did not avoid an excessive glycemic excursion, at least in some patients. The percentage of time spent in target range is higher in our study compared with other CL studies. In fact, a very recently published multicenter outpatient study in close supervision (hotel, guest house, hybrid hospital-hotel) reported $72 \%$ of the overnight time to be within $3.9-10 \mathrm{mmol} / \mathrm{L}^{24}$ Previous in-clinic CL trials using controllers that imitate the reasoning of diabetes caregivers ${ }^{15,25}$ have shown an increase in overnight glucose stability. However, CL comparison was done with CGM and not with venous glucose values.

In our study, reduction of hypoglycemia, measured as time spent with a BG level $<3.9 \mathrm{mmol} / \mathrm{L}$ and as reduction in the number of episodes under the same threshold, is significant and better than that observed in other single-hormone CL studies. $8,9,11$

Because the nocturnal period corresponds roughly with one-third of the total lifetime, near-normalization of glucose values during the night without increasing hypoglycemia risk would have a worthwhile impact on the diabetes burden.

Although our postprandial results were not exceptionally good, the use of the algorithm allows for a more predictable glucose excursion with a maximum around $90 \mathrm{~min}$ after meal intake. Knowing this could be useful for future control strategies.

The strategy used by our controller is different from the methods used to build an artificial pancreas most widely reported in the literature (model predictive control and proportional integral derivative). However, solutions based on rule-based approaches have also yielded improvement in glycemic control, ${ }^{15}$ as confirmed by our experience with the pRBA controller. Despite the increasing number of clinical studies to test alternative control algorithms, it is unclear whether any one system has more advantages than another.

Personalization in line with each patient's characteristics is an essential element to arrive at a successful algorithm. Model-based algorithms appear to be the most widely accepted strategy, as they consider physiologic knowledge and personal conditions by ad hoc adjustment of several parameters to each patient. Even so, setting a specific patient's model is quite a complex process. The pRBA algorithm is also adjusted to each patient by using the previously prescribed CSII therapy and personalized parameters, complemented by a generic predictive neural network trained with a population of T1DM patients, which makes generalization to a wider set of patients easier. ${ }^{18}$ It requires no previous training with each 


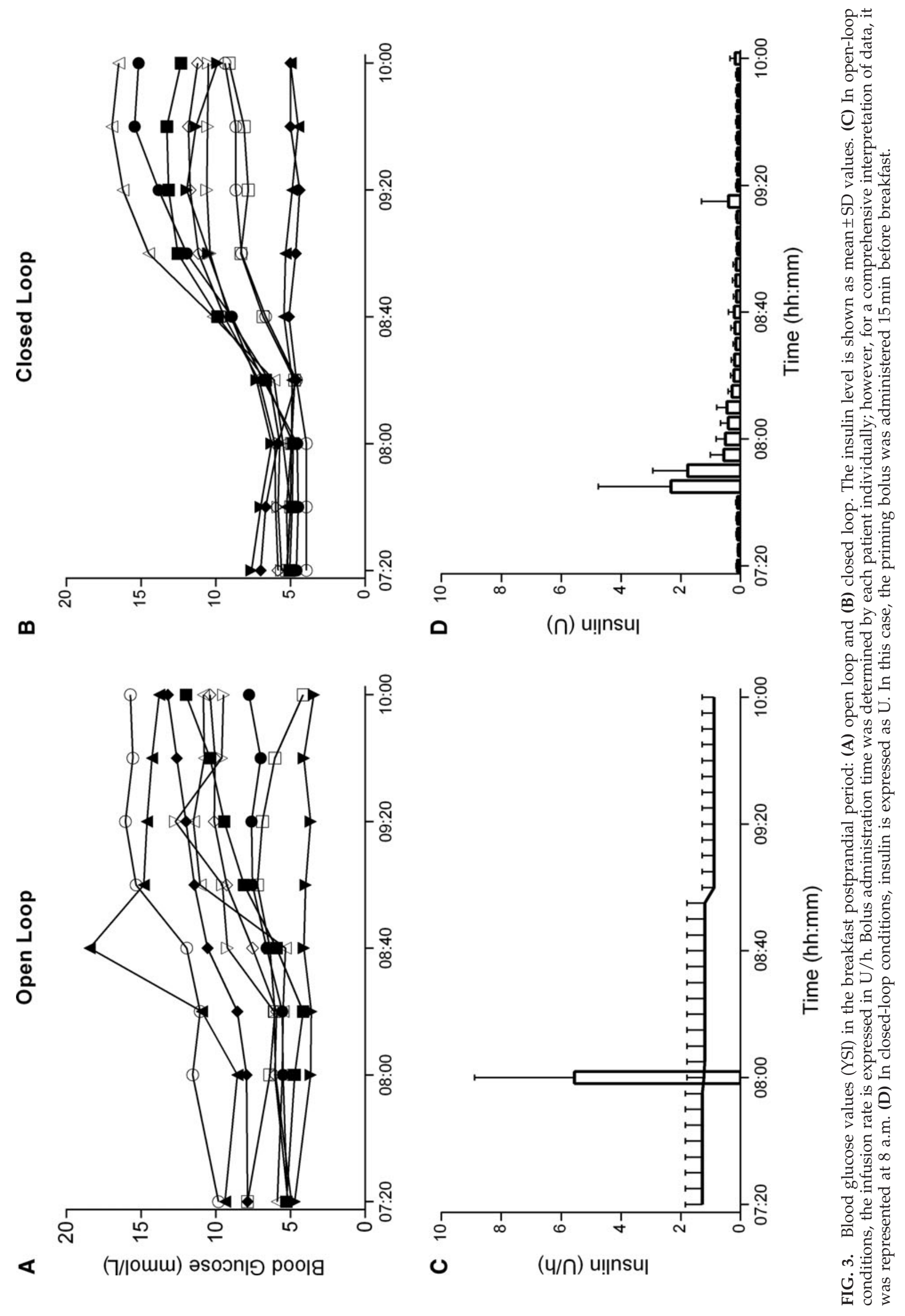


patient's specific features. The idea of predicting either hypoglycemia or hyperglycemia episodes as a safety alternative has been proposed previously. ${ }^{26}$ Our approach is not only to take advantage of glucose prediction to estimate the future rate of change in glucose levels, which was used by the pRBA as a safety constraint, but also to determine the accepted range in glucose stability.

As mentioned earlier, an unexpected degree of glucose stability was observed under CL conditions. We hypothesized that our algorithm proposing a microbolus every $5 \mathrm{~min}$, mimicking the physiological pulsatile insulin secretion, ${ }^{27,28}$ could contribute to insulin action optimization and favor stability. This pulsatile administration is different than the strategy used in other CL studies, ${ }^{7-9,13,15,29}$ in which a change in the insulin delivery rate is made so insulin is distributed throughout the actuation period.

Our study has some limitations. The sample size is small, although similar in number to other previously published CL studies. However, because the entire study was carried out at the same center, the risk of heterogeneity in procedures that can occur in multicenter trials is reduced. Communication between devices was done manually and could therefore be a source of errors, but stringent measures were taken to minimize the risk of error, and no omissions or discrepancies were observed between insulin doses proposed by the algorithm and those really administered. Two CGM devices were used: one as a reference for the experiment, whereas the second was kept as backup to be used in case of failure of the reference sensor. An outpatient study should address the management of the automatic detection of sensor failure. Future outpatient studies would require CGM calibration equal to that achieved by YSI to guarantee accuracy. The period between the breakfast and the end of the trial was too short, and probably a longer period of around 5-6h would have clarified whether the pRBA had any beneficial impact on the postprandial glycemic control.

In conclusion, this study shows that the pRBA is a new, more physiological, and highly precise controller that achieves a significant increase in overnight normoglycemia and glucose stability in patients with a previously acceptable metabolic control.

\section{Acknowledgments}

This work was funded by a grant from the Carlos III Health Institute (ISCIII 09/01255, 09/01318) and by the Parc Tauli Foundation (Parc Tauli Research Grants). Novolab Iberica S.A.L. (Madrid, Spain) provided the study insulin pumps. The authors wish to thank the patients for their enthusiastic participation in this trial.

\section{Author Disclosure Statement}

M.R. has received speaker honoraria from Medtronic and Roche, has served as a consultant to Novo Nordisk, and has received a grant from Roche Diagnostics. I.C., G.G.-S., A.R.-H., B.P., D.S., F.G.-G., M.G., M.A., C.P.-G., E.J.G., A.C., and M.E.H. declare no competing financial interests.

\section{References}

1. The effect of intensive treatment of diabetes on the development and progression of long-term complications in insulin-dependent diabetes mellitus. The Diabetes Control and Complications Trial Research Group. N Engl J Med 1993;329:977-986.

2. Hypoglycemia in the Diabetes Control and Complications Trial. The Diabetes Control and Complications Trial Research Group. Diabetes 1997;46:271-286.

3. van Belle TL, Coppieters KT, von Herrath MG: Type 1 diabetes: etiology, immunology, and therapeutic strategies. Physiol Rev 2011;91:79-118.

4. Yeh HC, Brown TT, Maruthur N, Ranasinghe P, Berger Z, Suh YD, Wilson LM, Haberl EB, Brick J, Bass EB, Golden SH: Comparative effectiveness and safety of methods of insulin delivery and glucose monitoring for diabetes mellitus: a systematic review and meta-analysis. Ann Intern Med 2012; 157:336-347.

5. Bellazzi R, Nucci G, Cobelli C: The subcutaneous route to insulin-dependent diabetes therapy. IEEE Eng Med Biol Mag 2001;20:54-64.

6. Steil GM, Rebrin K, Darwin C, Hariri F, Saad MF: Feasibility of automating insulin delivery for the treatment of type 1 diabetes. Diabetes 2006;55:3344-3350.

7. O'Grady MJ, Retterath AJ, Keenan DB, Kurtz N, Cantwell M, Spital G, Kremliovsky MN, Roy A, Davis EA, Jones TW, Ly TT: The use of an automated, portable glucose control system for overnight glucose control in adolescents and young adults with type 1 diabetes. Diabetes Care 2012;35: 2182-2187.

8. Hovorka R, Allen JM, Elleri D, Chassin LJ, Harris J, Xing D, Kollman C, Hovorka T, Larsen AM, Nodale M, De Palma A, Wilinska ME, Acerini CL, Dunger DB: Manual closed-loop insulin delivery in children and adolescents with type 1 diabetes: a phase 2 randomised crossover trial. Lancet 2010; 375:743-751.

9. Breton M, Farret A, Bruttomesso D, Anderson S, Magni L, Patek S, Dalla Man C, Place J, Demartini S, Del Favero S, Toffanin C, Hughes-Karvetski C, Dassau E, Zisser H, Doyle FJ $3^{\text {rd }}$, De Nicolao G, Avogaro A, Cobelli C, Renard E, Kovatchev B; International Artificial Pancreas Study Group: Fully integrated artificial pancreas in type 1 diabetes: modular closed-loop glucose control maintains near normoglycemia. Diabetes 2012;61:2230-2237.

10. Rodríguez-Herrero A, Pérez-Gandía C, Rigla M, de Leiva A, Gómez EJ, Hernando ME: A simulation study of an inverse controller for closed- and semiclosed-loop control in type 1 diabetes. Diabetes Technol Ther 2010;12:95-104.

11. Phillip M, Battelino T, Atlas E, Kordonouri O, Bratina N, Miller S, Biester T, Stefanija MA, Muller I, Nimri R, Danne T: Nocturnal glucose control with an artificial pancreas at a diabetes camp. N Engl J Med 2013;368:824-833.

12. Wang $\mathrm{Y}$, Dassau E, Zisser H, Jovanovič L, Doyle FJ $3^{\text {rd: }}$ Automatic bolus and adaptive basal algorithm for the artificial pancreatic $\beta$-cell. Diabetes Technol Ther 2010;12:879887.

13. Elleri D, Allen JM, Kumareswaran K, Leelarathna L, Nodale M, Caldwell K, Cheng P, Kollman C, Haidar A, Murphy HR, Wilinska ME, Acerini CL, Dunger DB, Hovorka R: Closedloop basal insulin delivery over 36 hours in adolescents with type 1 diabetes: randomized clinical trial. Diabetes Care 2013;36:838-844.

14. Renard E, Place J, Cantwell M, Chevassus H, Palerm CC: Closed-loop insulin delivery using a subcutaneous glucose sensor and intraperitoneal insulin delivery: feasibility study testing a new model for the artificial pancreas. Diabetes Care 2010;33:121-127. 
15. Atlas E, Nimri R, Miller S, Grunberg EA, Phillip M: MDLogic artificial pancreas system: a pilot study in adults with type 1 diabetes. Diabetes Care 2010;33:1072-1076.

16. Clarke WL, Anderson S, Breton M, Patek S, Kashmer L, Kovatchev B: Closed-loop artificial pancreas using subcutaneous glucose sensing and insulin delivery and a model predictive control algorithm: the Virginia experience. J Diabetes Sci Technol 2009;3:1031-1038.

17. Patek SD, Magni L, Dassau E, Karvetski C, Toffanin C, De Nicolao G, Del Favero S, Breton M, Man CD, Renard E, Zisser $\mathrm{H}$, Doyle FJ $3^{\text {rd }}$, Cobelli C, Kovatchev BP; International Artificial Pancreas (iAP) Study Group: Modular closed-loop control of diabetes. IEEE Trans Biomed Eng 2012;59:2986-2999.

18. Pérez-Gandía C, Facchinetti A, Sparacino G, Cobelli C, Gómez EJ, Rigla M, de Leiva A, Hernando ME: Artificial neural network algorithm for online glucose prediction from continuous glucose monitoring. Diabetes Technol Ther 2010; 12:81-88.

19. Hovorka R, Canonico V, Chassin LJ, Haueter U, MassiBenedetti M, Orsini Federici M, Pieber TR, Schaller HC, Schaupp L, Vering T, Wilinska ME: Nonlinear model predictive control of glucose concentration in subjects with type 1 diabetes. Physiol Meas 2004;25:905-920.

20. Kovatchev BP, Cox DJ, Gonder-Frederick LA, YoungHyman D, Schlundt D, Clarke W: Assessment of risk for severe hypoglycemia among adults with IDDM: validation of the Low Blood Glucose Index. Diabetes Care 1998; 21:1870-1875.

21. Clarke WL, Cox D, Gonder-Frederick LA, Carter W, Pohl SL: Evaluating clinical accuracy of systems for self-monitoring of blood glucose. Diabetes Care 1987;10:622-628.

22. Kovatchev BP, Gonder-Frederick LA, Cox DJ, Clarke WL: Evaluating the accuracy of continuous glucose-monitoring sensors: continuous glucose-error grid analysis illustrated by TheraSense Freestyle Navigator data. Diabetes Care 2004;27: 1922-1928.

23. Magni L, Raimondo DM, Man CD, Breton M, Patek S, Nicolao GD, Cobelli C, Kovatchev BP: Evaluating the efficacy of closed-loop glucose regulation via control-variability grid analysis. J Diabetes Sci Technol 2008;2:630-635.

24. Kovatchev BP, Renard E, Cobelli C, Zisser HC, Keith-Hynes $\mathrm{P}$, Anderson SM, Brown SA, Chernavvsky DR, Breton MD, Farret A, Pelletier MJ, Place J, Bruttomesso D, Del Favero S, Visentin R, Filippi A, Scotton R, Avogaro A, Doyle FJ $3^{\text {rd: }}$ Feasibility of outpatient fully integrated closed-loop control: first studies of wearable artificial pancreas. Diabetes Care 2013;36:1851-1858.

25. Nimri R, Danne T, Kordonouri O, Atlas E, Bratina N, Biester T, Avbelj M, Miller S, Muller I, Phillip M, Battelino T: The "Glucositter" overnight automated closed loop system for type 1 diabetes: a randomized crossover trial. Pediatr Diabetes 2013;14:159-167.

26. Dassau E, Cameron F, Lee H, Bequette BW, Zisser H, Jovanovic L, Chase HP, Wilson DM, Buckingham BA, Doyle FJ $3^{\text {rd }}$ : Real-time hypoglycemia prediction suite using continuous glucose monitoring: a safety net for the artificial pancreas. Diabetes Care 2010;33:1249-1254.

27. Hellman B: Pulsatility of insulin release-a clinically important phenomenon. Ups J Med Sci 2009;114:193-205.

28. Wahren J, Kallas A: Loss of pulsatile insulin secretion: a factor in the pathogenesis of type 2 diabetes? Diabetes 2012;61:2228-2229.

29. Dassau E, Zisser H, Harvey RA, Percival MW, Grosman B, Bevier W, Atlas E, Miller S, Nimri R, Jovanovic L, Doyle FJ $3^{\text {rd: }}$ Clinical evaluation of a personalized artificial pancreas. Diabetes Care 2013;36:801-809.

Address correspondence to: Ismael Capel, $\mathrm{MD}$ Endocrinology and Nutrition Department Parc Tauli Sabadell University Hospital Parc Taulí, s/n 08208 Sabadell-Barcelona, Spain E-mail: icapel@tauli.cat 